\title{
Correction to: ZNF326 promotes malignant phenotype of glioma by up-regulating HDAC7 expression and activating Wnt pathway
}

Xinmiao $\mathrm{Yu}^{1}$, Minghao Wang ${ }^{2 *}$, Jingjing $\mathrm{Wu}^{3}$, Qiang $\operatorname{Han}^{4}$ and Xiupeng Zhang ${ }^{4}$

\section{Correction to: J Exp Clin Cancer Res (2019) 38:40}

https://doi.org/10.1186/s13046-019-1031-4

In the original publication of this manuscript [1], the author mislabeled the CTL group and ZNF326 group in Fig.2-I,J (MTT result). The revised Fig. 2 is shown below.

The authors sincerely apologize for the inconvenience caused to the readers.

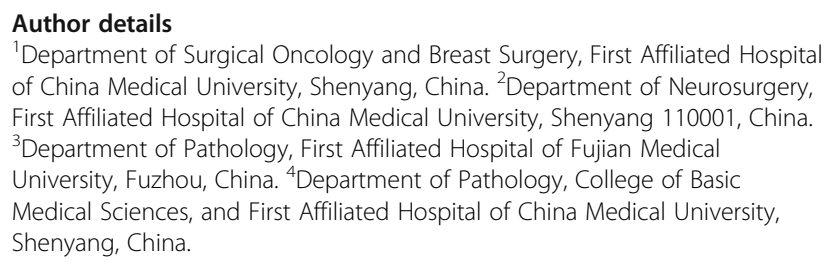

Published online: 16 January 2020

\section{Reference}

\footnotetext{
1. Yu X, et al. ZNF326 promotes malignant phenotype of glioma by upregulating HDAC7 expression and activating Wnt pathway. J Exp Clin Cancer Res. 2019;38:40. https://doi.org/10.1186/s13046-019-1031-4.
}

Full list of author information is available at the end of the article 
A

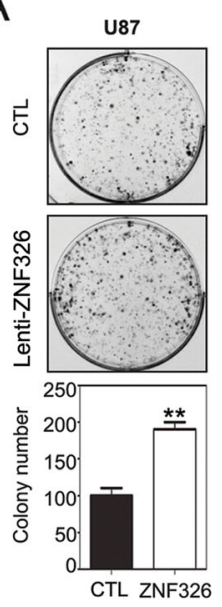

E
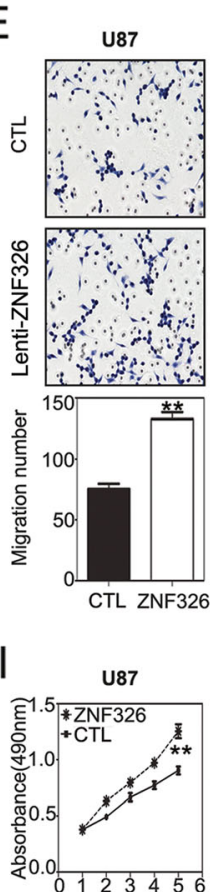

B

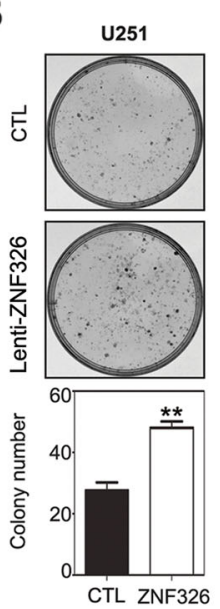

F
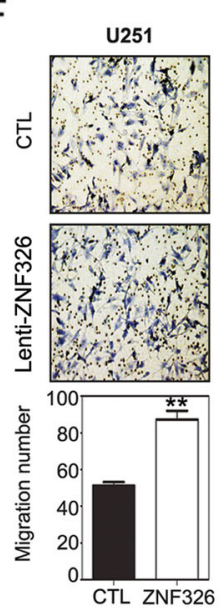

J

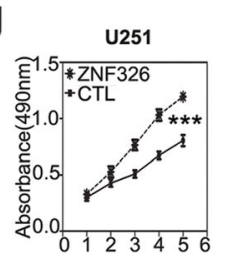

C

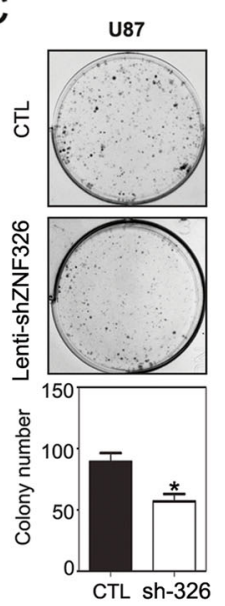

G

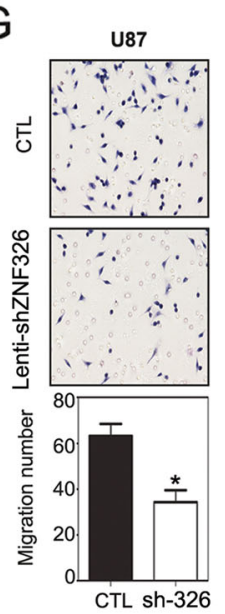

K

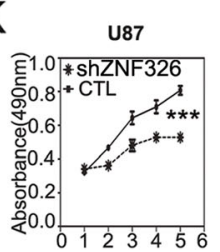

D

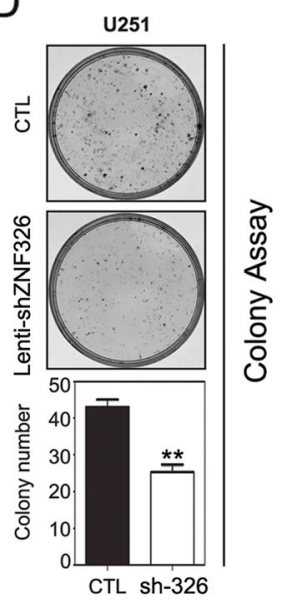

$\mathrm{H}$

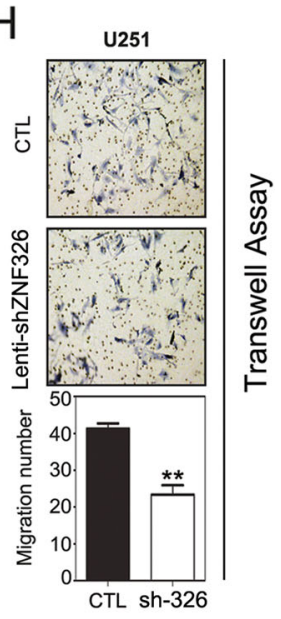

$\mathrm{L}$

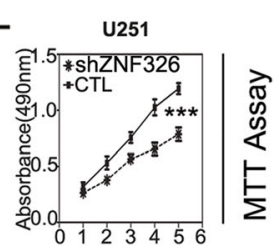

Fig. 2 Impact of ZNF326 expression on the proliferation and invasiveness of glioma cells in vitro. ZNF326 overexpression significantly enhanced the colony formation $(\mathbf{a}, \mathbf{b})$, invasiveness (e, $\mathbf{f}$, magnification-400x), and proliferation (i, j) of U87 and U251 glioma cell lines. Conversely, ZNF326 knockdown significantly inhibited colony formation (c, d), invasiveness ( $\mathbf{g}, \mathbf{h}$, magnification-400X), and proliferation ( $\mathbf{k}$, I) of U87 and U251 glioma

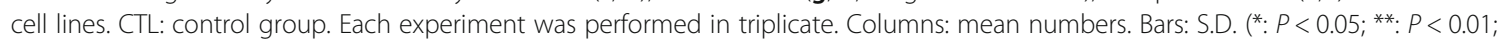

